\title{
Smart Roaming: How Operator Cooperation Can Increase Spectrum Usage Efficiency At Practically No Cost
}

\author{
by \\ Bharat Venkitesh \\ A thesis \\ presented to the University of Waterloo \\ in fulfillment of the \\ thesis requirement for the degree of \\ Master of Applied Science \\ in \\ Electrical and Computer Engineering
}

Waterloo, Ontario, Canada, 2018

(C) Bharat Venkitesh 2018 
I hereby declare that I am the sole author of this thesis. This is a true copy of the thesis, including any required final revisions, as accepted by my examiners.

I understand that my thesis may be made electronically available to the public. 


\begin{abstract}
We propose Smart Roaming (SR), a cooperation technique between cellular telcos operating in the same region, that enables users to roam for performance reasons (instead of lack of coverage from their operator). Within a region, base-stations of different operators are seldom co-located and even when they are, the sectors are rarely aligned. SR leverages spatial diversity to enhance spectrum usage efficiency, specifically an edge user of an operator might well be a "good user" for another one. This work answers the following research questions: i) Can significant gain be obtained with SR? ii) What are the factors that affect the gain? iii) How to deal with operator heterogeneity to avoid that a large operator cross subsidizes a smaller one? iv) How to implement SR in an online fashion?

We answer the first three questions by proposing a snapshot model for the downlink that shows that SR can indeed provide significant gain without yielding cross-subsidies if done properly. We then propose two schemes to implement SR online 1) a modification of the scheduler to allow base-stations to discriminate between users of different operators (a necessity to avoid cross-subsidy) jointly with a "free" user association (UA) whereby each user selects the best base-station irrespective of the operator it belongs to; 2) a controlled UA based on a distributed load sharing algorithm combined with the legacy scheduler. We evaluate these two schemes via extensive simulations based on two traffic scenarios and find gains in efficiency (defined as the per-operator sum-rate) above $25 \%$ and better performance for the first scheme. However, the second scheme might be easier to adopt since it does not affect the schedulers.
\end{abstract}




\section{Acknowledgements}

I would like to thank my supervisor Professor Catherine Rosenberg for her guidance and insightful advice.

I would also like to thank Professor Patrick Mitran and Professor Amir Khandani for their comments on the thesis. 


\section{Dedication}

This is dedicated to my parents and teachers. 


\section{Table of Contents}

List of Tables $\quad$ viii

List of Figures $\quad$ ix

List of Abbreviations $\quad$ x

List of Symbols $\quad$ xi

1 Introduction $\quad 1$

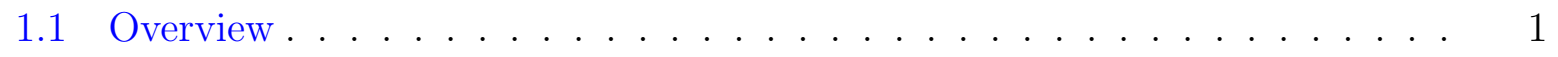

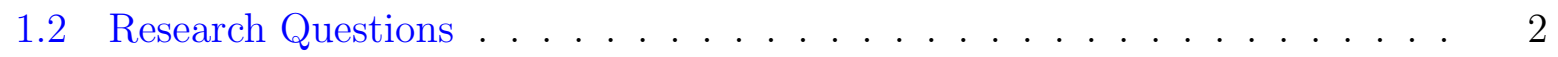

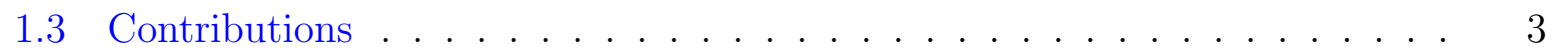

1.4 Literature Review . . . . . . . . . . . . . . . . . . . . 4

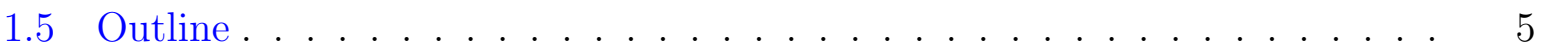

2 Smart Roaming : Static Case $\quad 7$

2.1 Introduction . . . . . . . . . . . . . . . . . 7

2.2 System Model . . . . . . . . . . . . . . . . . . . . . 8

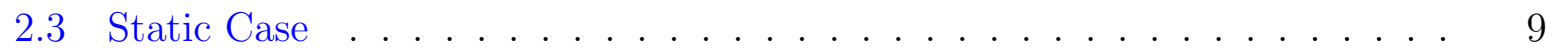


2.3 .1 Problem Formulations . . . . . . . . . . . . . . . . . . . 10

2.3 .2 Numerical Results . . . . . . . . . . . . . . . . . . . . . . . . . . . 12

2.3.3 Optimal and Heuristic Schedulers . . . . . . . . . . . . . 17

2.4 Conclusions . . . . . . . . . . . . . . . . . . . . . 20

3 Smart Roaming : Dynamic Case 21

3.1 Introduction . . . . . . . . . . . . . . . . . . . . . . . . . . . 21

3.2 Online Case . . . . . . . . . . . . . . . . . . . . . . . . . . . . . 22

3.2 .1 No Roaming $(\mathrm{NR}) \quad \ldots \ldots \ldots \ldots \ldots$

3.2 .2 Full Roaming $(\mathrm{FR}) \ldots \ldots \ldots$. . . . . . . . . . . . . . . 24

3.2.3 Smart Roaming 1 (SR1) scheme based on WRR . . . . . . . . . 24

3.2.4 Smart Roaming 2 (SR2) scheme based on load sharing $\ldots \ldots . .25$

3.2 .5 Numerical Results . . . . . . . . . . . . . . . . . . . . . 28

3.3 Conclusion . . . . . . . . . . . . . . . . . . . . . . . . 33

4 Conclusion and Future Work 34

4.1 Contributions . . . . . . . . . . . . . . . . . . . 34

4.2 Future Work . . . . . . . . . . . . . . . . . . . . . . 35

$\begin{array}{lr}\text { References } & 36\end{array}$ 


\section{List of Tables}

2.1 Physical Layer Parameters . . . . . . . . . . . . . . . . . . . 14 


\section{List of Figures}

2.1 Network topology . . . . . . . . . . . . . . . . . . . . . . . 14

2.2 Two-operator system: Average gain (over 100 realizations) as a function of $d=d_{x}=d_{y}$ and $\theta$ (Case 1$) \ldots \ldots \ldots \ldots$

2.3 Two-operator system: Average gain (over 100 realizations) as a function of $d=d_{x}=d_{y}$ and $\theta($ Case 2$) \ldots \ldots \ldots$. . . . . . . . . . . . . .

2.4 Average gain (over 100 realizations) in the three-operator system . . . . . . 17

2.5 Comparison of the GM throughput with the optimal scheduler and with WRR as a function of $N_{2}$ for the two-operator system (Case 2, $N_{1}=140$ ) . 18

2.6 Comparison of the GM throughput with the optimal scheduler and with WRR as a function of $N_{2}=N_{3}=N$ for the three-operator system . . . . . 20

3.1 Network architecture . . . . . . . . . . . . . . . . . 23

3.2 Scenario 1: Average download rate as a function of $\lambda_{2}, \lambda_{1}=1.25 \ldots$. . . . 30

3.3 Scenario 1: Average download rate as a function of $\lambda_{2}, \lambda_{1}=1.25 \ldots$. . . . 30

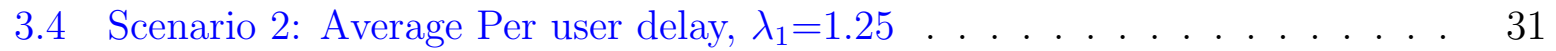

3.5 Scenario 2: Average Per user delay, $\lambda_{1}=1.25 \ldots \ldots$. . . . . . . . . . 32

3.6 Three operator case . . . . . . . . . . . . . . . . . 33 


\title{
List of Abbreviations
}

\author{
BTS Base Stations \\ SR Smart Roaming \\ UA User Association \\ US User Scheduling \\ WRR Weighted Round Robin \\ SINR Signal to Interference Noise Ratio \\ MINLP Mixed Integer Non Linear Programming \\ MCS Modulation and Coding Schemes \\ SR/EG Smart Roaming/Equal Gain \\ NR No Roaming \\ FR Full Roaming
}




\title{
List of Symbols
}

\author{
$Q \quad$ Set of Operators \\ $q \quad$ Operator \\ $Z_{q} \quad$ Set of sectors of Operator $q$ \\ $M_{q} \quad$ Sub channels of Operator $q$ \\ $P^{B T S} \quad$ Total transmit power of BTS \\ $\mathcal{U}_{q} \quad$ Number of users of Operator $q$ \\ $G_{i, j}^{c} \quad$ Channel gains for user $i$ in sector $j$ \\ $\gamma_{i, j}^{c} \quad$ SINR for user $i$ in sector $j$ \\ $R_{i, j} \quad$ Link rate for user $i$ in sector $j$ \\ $\lambda_{i, j} \quad$ Throughput for user $i$ in sector $j$ \\ $\lambda_{i} \quad$ Throughput for user $i$ \\ $\Omega \quad$ Set of realizations \\ $\omega \quad$ One realization \\ $\Gamma_{q}^{(0)} \quad$ GM throughput of Operator $q$ in NR \\ $\Gamma_{q}^{(1)} \quad$ GM throughput of Operator $q$ in SR/EG \\ $D_{i, j}\left(\phi_{i, j}\right) \quad$ Directivity gain for user $i$ in sector $j$ \\ $G^{a} \quad$ Antenna Gains \\ $\zeta_{i, j}^{c} \quad$ Slow Fading \\ $\rho_{q} \quad$ Gain in GM throughput of operator $q$ \\ $N_{q} \quad$ Total number of users of Operator $q$ \\ $n_{q j} \quad$ Number of users of operator $q$ in sector $j$ \\ $n_{j} \quad$ Number of users in sector $j$
}


$\alpha_{q j} \quad$ Fraction of time given to users of operator $q$

$U_{q}(t)$ Total number of bits transmitted to all users associated with sectors in $Z_{q}$

$L_{q}(t)$ Total number of bits received by all users of operator

$q$

$S_{q}(t) \quad$ Surplus load of operator $q$

$\Delta(T)$ Cumulative average surplus after $T$ minutes

$\tau \quad$ Threshold 


\section{Chapter 1}

\section{Introduction}

\subsection{Overview}

To meet the increased demand in mobile data [6], cellular operators are exploring new spectrum, e.g., mm-waves [22] as well as techniques to improve the efficiency of the usage of the spectrum they are already using. Such techniques range from physical layer technique such as MIMO technologies [16], to architectural enhancements such as heterogeneous cellular networks (HetNets) [21] that provide better reuse factor. However, these techniques increase Capital (CAPEX) and operational expenditures (OPEX) and the complexity of the network. Efficient spectrum sharing has proven to increase network capacity alleviating irregular load surges and improving the end user experience [25]. Hence, there is an increased interest by both operators and governing bodies like FCC to find ways to share resources efficiently [5], [11], [18]. Inter-operator resource sharing has been mostly limited to physical infrastructure [10], [17] and spectrum sharing [25]. One resource that has not been considered much in this context are the users.

Smart Roaming (SR) is about allowing users to be exchanged between operators covering the same region for efficiency reasons. SR is an inter-operator cooperation technique that is about user sharing, uses existing signaling mechanisms making it a practically costfree technique, is simple, scalable and cost effective. Within a region, base-stations (BTSs) 
of different operators are seldom co-located ${ }^{1}$ and even when they are, the sectors are rarely aligned [2]. SR leverages spatial diversity to enhance spectrum usage efficiency, specifically an edge user of an operator might well be a "good user" for another one. Exchanging such edge users will benefit everyone, though it has to be done in a way that does not create cross-subsidies, i.e., to avoid that one operator gains more than the others. Indeed, operators are heterogeneous, meaning they could differ in the number of BTSs, sub-channels as well as average number of users per subchannel and BTS which can be seen as a rough estimate of load. SR would require the cooperation of the operators, i.e., the sharing of information and the modification of their user association (UA) schemes as well as maybe other resource management processes such as user scheduling (US).

UA is the process of selecting a BTS for a user. A typical UA scheme assigns a user to the BTS that offers the best signal to interference plus noise ratio (SINR). US on the downlink is the quasi real-time local process that allocates resource blocks (RBs) and power to the transmissions to users associated to a particular BTS. A well known scheduling policy is based on equal power, i.e., the BTS uses the same power on all RBs and round robin (RR), i.e., equal number of RBs are allocated to all the users associated to a BTS. At first glance it is natural to try to implement SR by modifying and coordinating UA between operators without any changes in US, similar to the one proposed in [9].

\subsection{Research Questions}

Specifically, this work answers the following research questions:

1. Can significant gain be obtained with SR?

2. What are the factors that affect the gain?

3. How to deal with operator heterogeneity to avoid that a large operator cross subsidizes a smaller one?

\footnotetext{
${ }^{1}[14]$ says that only $10 \%$ of the BTSs are co-located in the UK and Poland.
} 
4. How to implement SR in an online fashion?

We answer the first three questions by proposing a snapshot model for the downlink that shows that SR can indeed provide significant gain without yielding cross-subsidies if done properly. We then show how to implement SR online by one of the following two schemes: 1) a modification of the scheduler to allow base-stations to discriminate between users of different operators (a necessity to avoid cross-subsidy) jointly with a "free" user association (UA) whereby each user selects the best base-station irrespective of the operator it belongs to; 2) a controlled UA based on a distributed load sharing algorithm combined with the legacy scheduler, i.e., no modification of the scheduling.

\subsection{Contributions}

The contributions of this work are as follows.

1. We propose a snapshot model for evaluating the performance of SR on the downlink when we enforce equal gain and compare its performance with the benchmark where each operator works independently and only accept its own users. This model jointly optimizes UA and US. Through extensive computations for different systems (e.g., 2 operators, 3 operators, different topologies), we show that the gains are significant.

2. We propose a weighted round robin (WRR) heuristic scheduler to replace the legacy RR scheduler in all the BTSs and show that it is quasi optimal in scenarios with 2 and 3 operators. In WRR, all users from the same operator receives the same amount of resource blocks and we show how to compute the ratio of these amounts.

3. We then propose and evaluate two schemes to implement SR online for the two operator case: Scheme 1: Any arriving user is free to choose the best BTS from either operator and each BTS in the region uses a WRR scheduler. Scheme 2: UA is controlled to provide load sharing via a distributed algorithm that we design and 
each BTS uses its legacy scheduler. We discuss for each scheme the information to be exchanged by the two operators.

The main message is that SR can bring significant gains with little associated cost even when the BTSs of the different operators are co-located as long as the sectors are not aligned. The gains in sum throughput can be as large as $29 \%$ for the case with 2 operators and $43 \%$ for the case with 3 operators. The most efficient way to implement SR is by using Scheme 1 where each BTS changes its scheduler from RR to WRR. We believe that the results are so encouraging that bodies such as the FCC might choose to mandate SR in the future to ensure better spectrum usage efficiency.

\subsection{Literature Review}

Several techniques have been proposed to improve spectrum usage, for example via massive MIMO [16], heterogeneous cellular networks (HetNets) [21] etc. In HetNEts the use of many different types of BTSs, e.g., macro, pico and femto, improves the reuse factor and hence the data rates. New ways to share the spectrum have been proposed in several EU projects such as METIS [7] and SAPHYRE [13]. Spectrum sharing has proven to improve the network efficiency for cellular operators. In [25], the authors propose to allocate frequency bands dynamically to operators and show that there is a significant gain in throughput by doing so. In this study we consider another resource to share, the users and propose solutions to efficiently share users between operators for performance reasons.

Traditional roaming is a type of user sharing in regions where a user cannot get service from its own operator. It is a well established method used by operators for increasing coverage. In the literature there are a few notable works that propose to share users between operators for performance reasons. In [20], the authors compare different options for inter-operator resource sharing, including what they call capacity sharing (CS) which is a generalization of traditional roaming. They propose an elaborate simulation framework to compare the performance of the sharing methods in a two operator system under moderate 
and heavy loading scenarios in co-located and non co-located topologies. Based on their simulation results, the authors report that CS is the best inter-operator sharing option in LTE cellular networks. Their work has no analytical results and focuses only on UA, i.e., the scheduling is unchanged. They also do not discuss the important problem of crosssubsidies. In our work, we propose analytical models and solutions based on both UA and scheduling that aim at minimizing cross-subsidies. In [9], the authors study user exchange in a two operator system, where they propose a scheme to swap users between operators. The authors impose that the exchanged users should not do worse than before. They first study the scheme in a centralized framework in which a central controller decides which users need to be swapped so that the number of users that each operator serves remains the same. They also propose a distributed swapping scheme, where users are swapped between pairs of BTSs. They report results for a system where BTSs are equipped with omni directional antennas and show that there is a significant gain in throughput for the edge users. Further, the authors show that the performance improves with increased spatial diversity between the BTSs of operators. In this work, we study sectored BTSs and propose a distributed solution in which we replace user swapping by load sharing. We also show the importance of scheduling and quantify the gains of our smart roaming solutions for 2 and 3 operator system.

\subsection{Outline}

The thesis is organized as follows,

In Chapter 2 we propose an snapshot model for evaluating the performance of SR on the downlink by jointly optimizing UA and uS. We quantify the gains on different systems (e.g. 2, 3 operators, multiple topologies). We also propose a weighted round robin (WRR) heuristic scheduler and show that it is quasi optimal. In WRR, all users from the same

operator receives the same amount of resource blocks and we discuss how to compute the ratio of these amounts.

In Chapter 3 we extend our analysis to dynamic case and propose two online schemes 
to implement SR. Through simulations we show that a simple scheme with a free UA and RR scheduling yields unacceptable cross subsidies. We show that a scheme based on free UA and modifying the legacy scheduler to WRR performs better than a scheme that only controls UA with RR scheduling.

Chapter 4 reviews conclusions drawn from SR in both static and dynamic case and discusses the avenues for future work. 


\section{Chapter 2}

\section{Smart Roaming : Static Case}

Summary: In this chapter, we

- Propose a snapshot model for evaluating the performance of SR on the downlink by jointly optimizing UA and US,

- Quantify the gains for our model on 2 and 3 operators and for various topologies

- Propose a weighted round robin (WRR) heuristic scheduler and show that it is quasi optimal

\section{$2.1 \quad$ Introduction}

We start off with a snapshot model which is a simple, yet insightful, tool that enables us to formulate many network utility maximization problems and study the mean performance of the system by averaging the network utilities over a large number of realizations. In this chapter we study static setting, where the number of users are fixed and are not mobile. 


\subsection{System Model}

Consider a region where several operators have deployed their BTSs. Let $Q$ denote the set of operators. We consider the case where the BTSs are sectored. For each BTS we assume there are three non overlapping sectors. Let $\mathcal{Z}_{q}$ denote the set of sectors of operator $q \in Q$ (we do not assume that each operator has the same number of BTSs) and $\mathcal{Z}=\cup_{q} \mathcal{Z}_{q}$. We focus on the downlink. We make the following assumptions on each operator $q \in Q$.

- The network corresponding to operator $q$ is OFDMA-based and has been licensed $M_{q}$ sub-channels, each of bandwidth b. The reuse factor is 1 across sectors, i.e., all sectors of an operator are using the $M_{q}$ sub-channels.

- Each user equipment (UE) can associate with only one sector and all the users are greedy, i.e., they want the best possible throughput.

- All BTSs are always active, i.e., there is no instance when a BTS is not transmitting and a BTS uses all its transmit power at all time.

- The total transmit power of a BTS is $P_{B T S}$ and it is divided equally among its sector and then each sector divides its power equally among its sub-channels. Let $P^{c}$ be the transmit power per sub-channel in a sector, we have $P^{c}=\frac{P_{B T S}}{3 M_{q}}$.

- Let $\mathcal{U}_{q}(t)$ be the set of active UEs for operator $q$ at time $t$ and let $\mathcal{U}(t)$ be the total set of UEs over all operators. At any point of time $t$ the channel gains $G_{i, j}^{c}(t)$ on sub-channel $c$ from all (UE, sector) pair $(i, j)$ (with $i \in \mathcal{U}(t)$ and $j \in \mathcal{Z}$ ) are known so that the SINR from each sector to each UE can be computed.

- The rate function $f($.$) for each sector is the same and is given, so that given an SINR,$ the per channel user's throughput can be computed.

- From the above assumptions, given power $P^{c}$ and channel gains $G_{i, j}^{c}(t)$, we can calculate the per channel SINR $\gamma_{i, j}^{c}(t)$ at time $t$ of each user $i \in \mathcal{U}_{q}$ from all sectors 
$j \in \mathcal{Z}_{q}$ and for all $q \in Q$.

$$
\gamma_{i, j}^{c}(t)=\frac{P^{c} G_{i, j}^{c}(t)}{N_{0}+\sum_{j^{\prime} \neq j, j^{\prime} \in \mathcal{Z}_{q}} P^{c} G_{i, j}^{c}(t)}
$$

- The channels are assumed flat, hence the superscript $c$ can be dropped and we use $\gamma_{i, j}(t)$ instead to denote the SINR.

- Let $x_{i, j}(t)$ be 1 if user $i \in \mathcal{U}$ is associated with sector $j \in \mathcal{Z}$ at time $t$ and let it be zero otherwise ( $i$ and $j$ do not have to belong to the same operator). We define $R_{i, j}(t)$ as the link rate (i.e., the maximum obtainable rate in absence of other users) a user $i$ obtains from sector $j \in \mathcal{Z}$ at time $t$ where,

$$
R_{i, j}(t)=M_{q} f\left(\gamma_{i, j}(t)\right) \text { if } j \in \mathcal{Z}_{q}, \forall q \in Q
$$

- Let $\lambda_{i, j}(t)$ denote user $i$ 's throughput obtained from sector $j \in \mathcal{Z}$ at time $t$ iff $i$ is associated to $j$. Let $\alpha_{i, j}(t)$ be the proportion of time allocated to user $i$ at time $t$ by BTS $j$, then we compute the throughput as follows,

$$
\lambda_{i, j}(t)=R_{i, j}(t) \alpha_{i, j}(t) x_{i, j}(t)
$$

Hence, user $i$ receives a throughput, $\lambda_{i}(t)=\Sigma_{j \in \mathcal{Z}} x_{i, j}(t) \lambda_{i, j}(t)$.

- We select proportional fairness (PF) as our objective function in all our optimization frameworks. In that case, the system performance metric for an operator $q$ that characterizes both efficiency and fairness is the geometric mean (GM) of its UE throughputs given as $\left.\Gamma_{q}(t)=\Pi_{i=\mathcal{U}_{q}} \lambda_{i}(t)\right)^{1 /\left|\mathcal{U}_{q}\right|}$. We will also give results in terms of the sum-rate since it is a metric operators are more familiar with. Note that maximizing the sum-rate is not an acceptable objective for fairness reasons.

\subsection{Static Case}

For a given system characterized by $Q$, the set of operators, $\mathcal{Z}$ the set of sectors for each operator, and the $M_{q}$ 's, we begin by considering a snapshot model, where the set of users per 
operator, $\mathcal{U}_{q}(t)$, and their channel gains are fixed and known and characterize a snapshot or realization $\omega$ of the system. In that case, we can remove the time index. We generate a set $\Omega$ of realizations and study the performance of the different scenarios averaged over all the realizations in $\Omega$. We start off by introducing our benchmark, where each operator works independently and users are not allowed to associate with BTSs from other operators and formulate the centralized joint user association and scheduling problem that allows us to compute for a given realization $\omega$, the per operator GM throughput $\Gamma_{q}^{(0)}(\omega)$. We call our benchmark as "No Roaming". We then focus on the smart roaming case where we allow users to associate with any sector but we enforce that the gain in GM throughput is equal for each operator to avoid cross-subsidies. We call this scenario "Smart Roaming/Equal Gain". We then compare the performance of the two scenarios for different systems (i.e., 2 or 3 operators, different BTS locations, etc.).

\subsubsection{Problem Formulations}

\section{No Roaming}

Recall that the users of operator $q$ can only associate with sectors of their own operator. In that case, we can compute the performance for each operator independently. For a given realization $\omega$, we want to compute the $x_{i, j}$ 's (the UA parameters) and the $\alpha_{i, j}$ 's (the US parameters) for each $i \in \mathcal{U}_{q}$ from all sectors $j \in \mathcal{Z}_{q}$ to maximize the GM throughput

$\Gamma_{q}^{(0)}$ (we omit the index $\omega$ for ease of notation). Note that maximizing $\Gamma_{q}^{(0)}$ is equivalent to maximizing $\Sigma_{i \in \mathcal{U}_{q}} \log \left(\lambda_{i}\right)$, where $\lambda_{i}=\Sigma_{j \in \mathcal{Z}_{q}} x_{i, j} \lambda_{i, j}$. This problem is an MINLP. We relax the integer variables $x_{i, j}$ 's and solve it by using commercial solver, Minos 5.51 [3], thus obtaining an upper bound on the GM throughput. We extract a feasible solution to the original problem from the solution of the relaxed problem by selecting for a given user the sector $j$ that yields the largest $R_{i, j} \times \alpha_{i, j}$. This technique was shown in [24] to provide a feasible solution whose GM throughput is very close to the upper bound. Hence, the 
problem for the "No Roaming" scenario for operator $q$ is written as follows,

$$
\begin{array}{ll}
\max _{\left\{x_{i, j}, \alpha_{i, j}\right\}} \Sigma_{i \in \mathcal{U}_{q}} \log \left(\lambda_{i}\right) & \\
\lambda_{i}=\Sigma_{j \in \mathcal{Z}_{q}} \alpha_{i, j} R_{i, j}, & \forall i \in \mathcal{U}_{q} \\
\Sigma_{i \in \mathcal{U}_{q}} \alpha_{i j}=1 & \forall j \in \mathcal{Z}_{q} \\
\alpha_{i j} \leq x_{i j} & \forall i \in \mathcal{U}_{q}, \forall j \in \mathcal{Z}_{q} \\
\Sigma_{j \in \mathcal{Z}_{q}} x_{i, j}=1, & \forall i \in \mathcal{U}_{q} \\
x_{i, j} \in\{0,1\}, & \forall i \in \mathcal{U}_{q}, \forall j \in \mathcal{Z}_{q} \\
\alpha_{i j} \geq 0, & \forall i \in \mathcal{U}_{q}, \forall j \in \mathcal{Z}_{q}
\end{array}
$$

Note that it was shown in [8] that this centralized problem can be decoupled into individual round robin (RR) local schedulers, i.e., $\alpha_{i, j}=\frac{1}{n_{j}}$ where $n_{j}$ is the number of users associated to sector $j$.

\section{Smart Roaming/Equal Gain}

In this scenario, UA can be done across operators and US is not restricted to RR, it is optimized (jointly with UA) such that the gains in GM throughput of each operator with respect to the benchmark are equal. For a given realization $\omega$, given the per operator benchmark performance $\left(\Gamma_{1}^{(0)}, \Gamma_{2}^{(0)}, \ldots, \Gamma_{|Q|}^{(0)}\right)$, we want to compute the $x_{i, j}$ 's (the UA parameters) and the $\alpha_{i, j}$ 's (the US parameters) for each $i \in \mathcal{U}$ from all sectors $j \in \mathcal{Z}$ to maximize the GM throughput $\Gamma_{1}^{(1)}$ subject to the equal gain constraints. The problem can be written 
as follows,

$$
\begin{aligned}
& \max _{\left(x_{i, j}\right),\left(\alpha_{i, j}\right)} \Sigma_{i \in \mathcal{U}_{1}} \log \left(\lambda_{i}\right) \\
& \lambda_{i}=\Sigma_{j \in \mathcal{Z}} \alpha_{i, j} R_{i, j} \quad \forall i \in \mathcal{U} \\
& \Sigma_{j \in \mathcal{Z}} x_{i, j}=1, \quad \forall i \in \mathcal{U} \\
& \Sigma_{i \in \mathcal{U}} \alpha_{i, j}=1, \quad \forall j \in \mathcal{Z} \\
& \alpha_{i j} \leq x_{i j}, \quad \forall i \in \mathcal{U}, \forall j \in \mathcal{Z} \\
& \frac{\left(\Pi_{i \in \mathcal{U}_{1}} \lambda_{i}\right)^{1 /\left|\mathcal{U}_{1}\right|}}{\Gamma_{1}^{(0)}}=\frac{\left(\Pi_{i \in \mathcal{U}_{q}} \lambda_{i}\right)^{1 /\left|\mathcal{U}_{q}\right|}}{\Gamma_{q}^{(0)}} \quad \forall q \in Q \\
& x_{i, j} \in\{0,1\} \quad \forall i \in \mathcal{U}, \forall j \in \mathcal{Z} \\
& \alpha_{i, j} \geq 0 \quad \forall i \in \mathcal{U}, \forall j \in \mathcal{Z}
\end{aligned}
$$

Note that we only maximize the GM throughput of operator $q=1$, but constraint (2.16) enforces equal gain in GM throughput for each operator.

This problem is also an MINLP and constraint (2.16) makes the problem non convex. Similar to NR, we relax the integer variables $x_{i, j}$ 's and solve this relaxed problem to find an upper bound using commercial solver Baron [1] that is capable of finding a global optimum for non convex optimization problems. We then use the same technique as for NR to obtain a feasible solution out of the solution of the relaxed problem and have checked that the corresponding GM is very close to the upper bound. Let the GM throughput of that feasible solution for each operator $q$ be $\Gamma_{q}^{(1)}$.

\subsubsection{Numerical Results}

We show numerical results first for the case with 2 operators $(|Q|=2)$ and then for the case with 3 operators. 


\section{Two-operator case}

Let's begin by describing our network topology (for the case with 2 operators) represented in Fig. 2.1. We assume that both operators have 7 BTSs. Each network has an inter-BTS distance (ISD) of $500 \mathrm{~m}$. Each sector of the second operator is rotated by $\theta$ degrees wrt the corresponding sector of operator 1. The BTSs of operator 2 are also shifted by two parameters $\left(d_{x}, d_{y}\right)$, i.e., if BTS $j$ of operator 1 is at point $(x, y)$, then BTS $j$ of operator 2 is at point $\left(x+d_{x}, y+d_{y}\right)$. If $d_{x}=d_{y}=0$, the BTSs are co-located.

A network topology is thus characterized by the three parameters $\left(d_{x}, d_{y}, \theta\right)$ and we will study the performance of SR as a function of these three parameters. In order to avoid corner cases we assume a wrap around model of deployment. We calculate the channel gain $G_{i, j}^{c}$ (in $\left.\mathrm{dB}\right)$ on channel $c$ from sector $j$ to $\mathrm{UE} i, j \in \mathcal{Z}$ using:

$$
G_{i, j}^{c}=-D_{i, j}\left(\phi_{i, j}\right)-A_{i}-A_{j}+\zeta_{i, j}^{c}+\nu+P L_{i, j}(L),
$$

where $D_{i, j}\left(\phi_{i, j}\right)=-\min \left\{12\left(\frac{\phi_{i, j}}{70^{0}}\right)^{2}, 20\right\} d B$ represents the directivity gain where $\phi_{i, j}$ is the angle made by the user $i$ with the broadside direction of the antenna from sector $j$ [4], $A_{i}$ and $A_{j}$ are the antenna gains (17dBi for BTSs and 0dBi for UEs), $\nu$ is the penetration loss and $P L_{i, j}(L)$ is the path loss with $L$ being the distance between user $i$ and sector $j$. We further apply, $\zeta_{i, j}^{c}$, a log normal shadowing [12] of $8 \mathrm{~dB}$ standard deviation. Table 2.1 provides the physical layer parameters. We assume the system uses adaptive modulation with discrete rates. Table II in [19], provides the mapping between SINR and link rate $(\mathrm{kb} / \mathrm{s})$ [19]. When a user $i$ has a SINR level between $l$ and $l+1$ in sector $j$, then the link rate $R_{i, j}$ the user will receive from sector $j$ is $e_{l}$, where $e_{l}$ is the link rate at level $l$ as given in Table II in [19].

A two operator system is defined by a given topology characterized by $\left(d_{x}, d_{y}, \theta\right)$, the number of sub channels per operator, $M_{1}, M_{2}$ and $N_{1}=\left|\mathcal{U}_{1}\right|, N_{2}=\left|\mathcal{U}_{2}\right|$, the numbers of users of each operator where we assume that users are uniformly distributed within the geographical area. Specifically, we consider two cases:

- Case 1: Both operators have the same number of sub channels $\left(M_{1}=M_{2}=99\right)$, $N_{1}=140$ and we vary $N_{2}$. 
- Case 2: The operators have $M_{1}=99$ and $M_{2}=66$ sub-channels respectively, $N_{1}=140$ and we vary $N_{2}$.

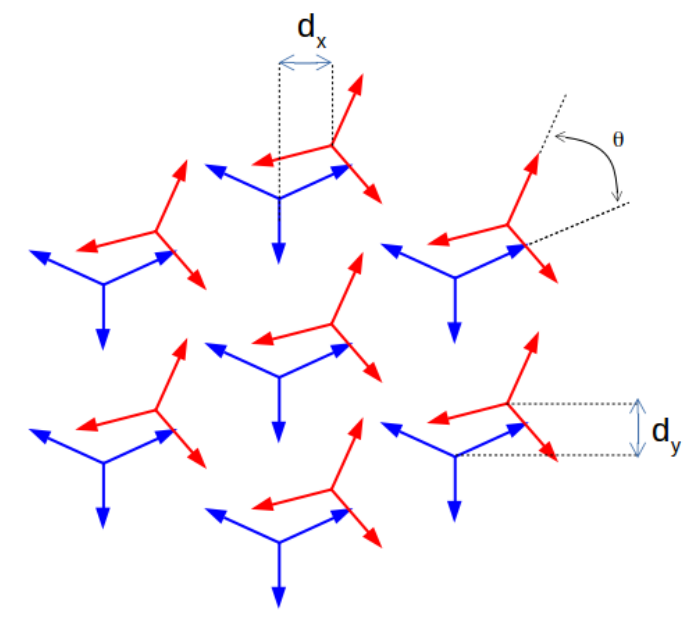

Figure 2.1: Network topology

Table 2.1: Physical Layer Parameters

\begin{tabular}{cccc}
\hline Noise Power & $-174^{\frac{d B m}{H z}}$ & $P^{B T S}$ & $46 \mathrm{dBm}$ \\
BTS antenna gain & $17 \mathrm{dBi}$ & $I S D$ & $500 \mathrm{~m}$ \\
UE antenna gain & $0 \mathrm{dBi}$ & $\nu$ & $20 \mathrm{~dB}$ \\
Channel bandwidth & $180 \mathrm{KHZ}$ & & \\
\hline$P L_{i, j}(L)=128+37.6 \log _{10}(L / 1000), L \geq 35$ \\
\hline
\end{tabular}

We then analyze the performance on numerous topologies by considering different values for $d=d_{x}=d_{y}$ and $\theta$, where $d \in\{0,10,20,30,40,50,60,70,80\}$ (in meters) and $\theta \in$ $\{0,15,30,45,60,75,90\}$ (in degrees). For each topology we compute the feasible solutions for the two problems: "No roaming (NR)" and "Smart Roaming/Equal gain (SR/EG)" described above. We evaluate the performance of SR/EG wrt NR via the per operator 
gain in GM defined as:

$$
\rho=\frac{\Gamma_{q}^{(1)}}{\Gamma_{q}^{(0)}} \quad \forall q \in Q
$$

where $\Gamma_{q}^{(1)}$ and $\Gamma_{q}^{(0)}$ are the GM throughputs for SR/EG and NR respectively. Note that by construction, the gain for each operator is the same for SR/EG. Figures 2.2 and 2.3 show the gain obtained by averaging over 100 realizations as a function of $(d, \theta)$ for Case 1 and Case 2 respectively and fixed values of $N_{1}$ and $N_{2}$.

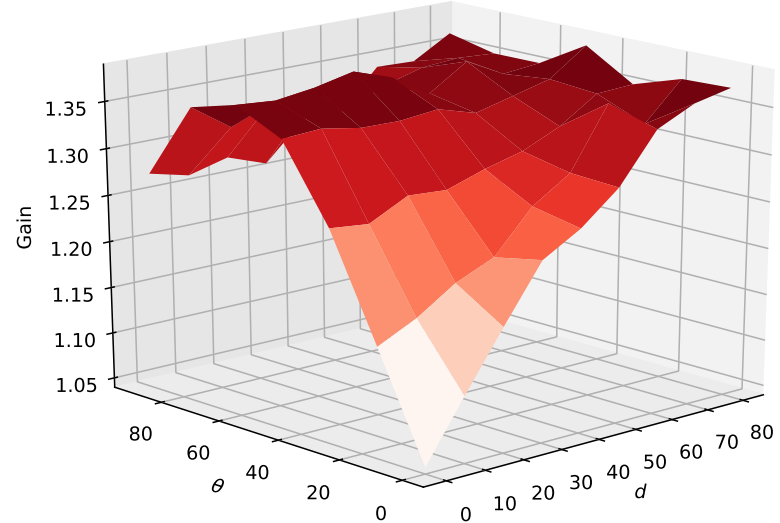

(a) $N_{1}=140$ and $N_{2}=105$

Figure 2.2: Two-operator system: Average gain (over 100 realizations) as a function of $d=d_{x}=d_{y}$ and $\theta$ (Case 1$)$ 


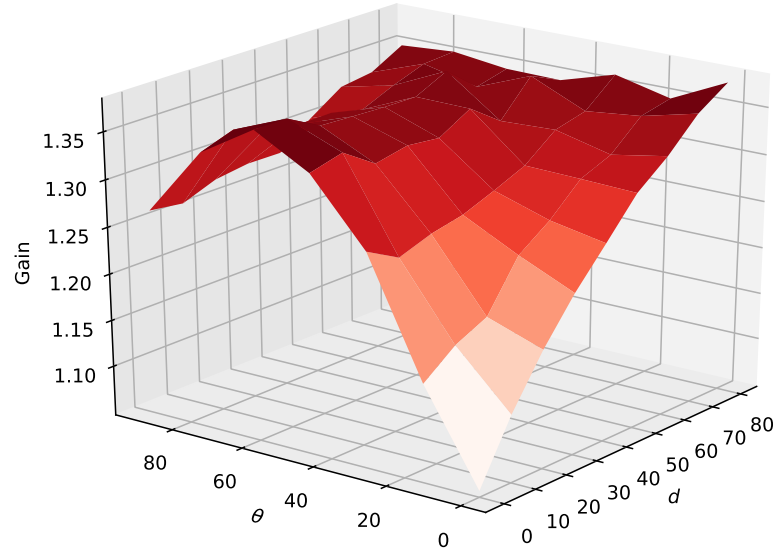

(a) $N_{1}=140$ and $N_{2}=140$

Figure 2.3: Two-operator system: Average gain (over 100 realizations) as a function of $d=d_{x}=d_{y}$ and $\theta$ (Case 2$)$

These figures show that SR/EG can achieve significant gains as long as there is enough diversity in the topologies. The gain in GM throughputs can be as high as $35 \%$ for certain configurations of the topology, even when the BTSs are co-located (this is the case for $\theta=60$ degrees). The corresponding gain in sum throughput is $29 \%$. The figures also show that a high gain can be obtained in an heterogeneous case such as Case 2 .

\section{Three-operator case}

Next we study the case of three operators where we assume that each operator has 7 colocated BTSs and, for simplicity, that each sector of the second operator is rotated by $\psi$ degrees wrt the corresponding sector of operator 1 and each sector of the third operator is rotated by $\psi$ degrees wrt the corresponding sector of operator 2 . We perform extensive computations for the following case: $M_{1}=99, M_{2}=M_{3}=66$ and $N_{1}=N_{2}=N_{3}=N$. We plot the gains (again averaged over 100 realizations) as a function of $\psi$ for three values of $N$ in Fig. 2.4. We obtain a stunning $53 \%$ gain in GM throughput when $\psi=40$ degrees. 
The corresponding gain in sum throughput is $43 \%$. Interestingly, we find that the gains are not sensitive to a large range of values of $N$. We saw similar results for the case of two operators.

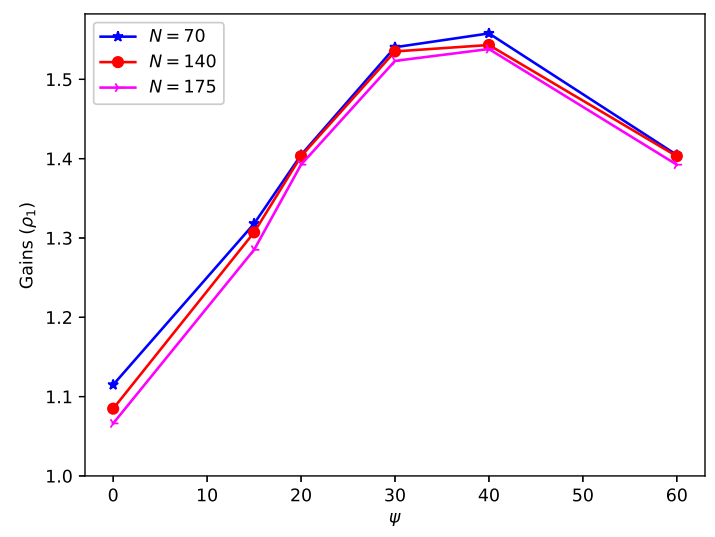

Figure 2.4: Average gain (over 100 realizations) in the three-operator system

These results show the high potential of smart roaming. Before addressing the online problem, i.e., how to design practical schemes that yield good performance, we investigate further the scheduling under SR/EG.

\subsubsection{Optimal and Heuristic Schedulers}

Note that there is no assumption on the scheduling in the SR/EG problem and our extensive computations (recall that each point of the figures above correspond to 100 realizations) have shown that the optimal scheduler is not $R R$ but provides practically equal time to users of the same operator, but different times to users from different operators. Based on this insight, we design a heuristic local scheduler for the two-operator system that we called "weighted round robin (WRR)" that we later generalize to the three-operator case. 


\section{Two-operator case:}

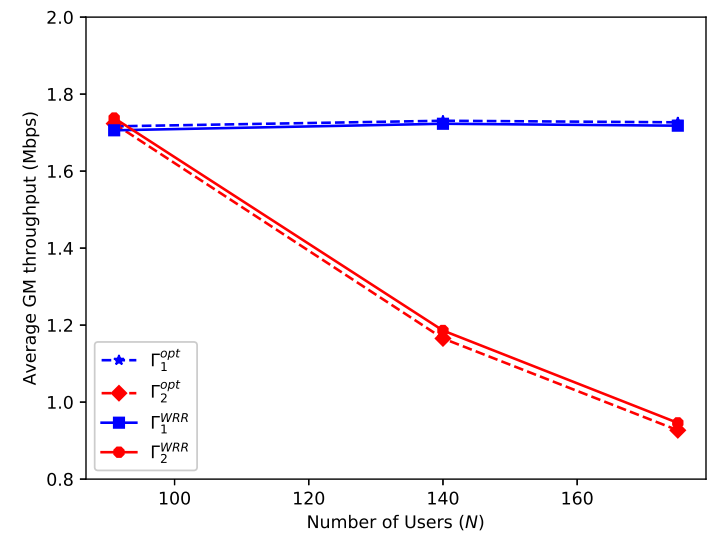

Figure 2.5: Comparison of the GM throughput with the optimal scheduler and with WRR as a function of $N_{2}$ for the two-operator system (Case $2, N_{1}=140$ )

Let us consider a sector $j \in \mathcal{Z}_{1} \cup \mathcal{Z}_{2}$. WRR offers the same fraction of time $\alpha_{1 j}$ (resp. $\alpha_{2 j}$ ) to all its associated users coming from operator 1 (resp. operator 2 ). We have

$$
n_{1 j} \alpha_{1 j}+n_{2 j} \alpha_{2 j}=1
$$

where $n_{1 j}$ (resp. $n_{2 j}$ ) is the number of users from operator 1 (resp. operator 2) associated with sector $j$. To compute $\alpha_{1 j}$ and $\alpha_{2 j}$, we need another equation which we derive heuristically by conjecturing as follows using a simple average argument.

Consider the "No Roaming" case and a sector of say operator 1. It would see on average $\frac{N_{1}}{\left|\mathcal{Z}_{1}\right|}$ users and a user $i \in \mathcal{U}_{1}$ would join the sector $j^{*} \in \mathcal{Z}_{1}$ yielding the highest $\operatorname{SINR} \gamma_{i, j^{*}}$. In that case, $i$ would see a throughput equal to $\frac{\left|\mathcal{Z}_{1}\right| M_{1}}{N_{1}} f\left(\gamma_{i, j^{*}}\right)$ (since the local scheduler is $\mathrm{RR}$ ). To avoid cross-subsidies, we have to make sure that the users from operator 1 do not roam because operator 2 provides a better "resource" ratio, i.e., because $\frac{\left|\mathcal{Z}_{1}\right| M_{1}}{N_{1}}<\frac{\left|\mathcal{Z}_{2}\right| M_{2}}{N_{2}}$ but because user $i$ sees a better SINR in one of the sectors of operator 2 , hence we enforce that:

$$
\frac{\alpha_{1 j}}{\alpha_{2 j}}=c=\frac{\left|\mathcal{Z}_{1}\right| M_{1} N_{2}}{N_{1}\left|\mathcal{Z}_{2}\right| M_{2}}
$$


since in that case, the only reason (under this average argument) to roam is to gain in SINR.

To take care of corner cases, we need to add that $c=0$ if $n_{1 j}=0$ and $c=1$ if $n_{2 j}=0$. Eqs. (2.21) and (2.22) along with the conditions for the corner cases define WRR.

To validate the accuracy of WRR by comparing the per operator GM throughput $\left(\Gamma_{q}^{W R R}\right)$ when using WRR instead of the optimal scheduler with $\left(\Gamma_{q}^{o p t}\right)$ over many realizations and many systems. Specifically, for each realization, we obtain the UA parameters, i.e., the $x_{i, j}$ 's from the feasible solution and then compute for each sector $j$, the WRR weights $\left(\alpha_{i, j}\right)$ using Eqs. (2.21) and (2.22) and then compute the corresponding GM throughput $\left(\Gamma_{q}^{W R R}\right)$ for each operator. We show the results (averaged over 100 realizations for each value of $N_{2}$ ) as a function of $N_{2}$ for the co-located system with $\theta=60$ for Case 2 when $N_{1}=140$ in Fig. 2.5. The difference in GM is never more than $1.3 \%$ which validate WRR as an excellent heuristic scheduler.

\section{Three-operator case:}

We now extend WRR by enforcing that:

$$
\begin{aligned}
& \frac{\alpha_{1 j}}{\alpha_{2 j}}=\frac{\left|\mathcal{Z}_{1}\right| M_{1} N_{2}}{N_{1}\left|\mathcal{Z}_{2}\right| M_{2}}, \quad \frac{\alpha_{1 j}}{\alpha_{3 j}}=\frac{\left|\mathcal{Z}_{1}\right| M_{1} N_{3}}{N_{1}\left|\mathcal{Z}_{3}\right| M_{3}} \\
& n_{1 j} \alpha_{1 j}+n_{2 j} \alpha_{2 j}++n_{3 j} \alpha_{3 j}=1
\end{aligned}
$$

Similar to the two-operator case, we compare the average GM throughput for WRR and the optimal scheduling. We show the result as a function of $N\left(N_{2}=N_{3}=N\right)$ for colocated BTSs and $\phi=40$ degrees when $N_{1}=140$ in Fig. 2.6. Again, the difference in GM is never more than $1.5 \%$ which validate WRR as an excellent heuristic scheduler in the case of three operators. 


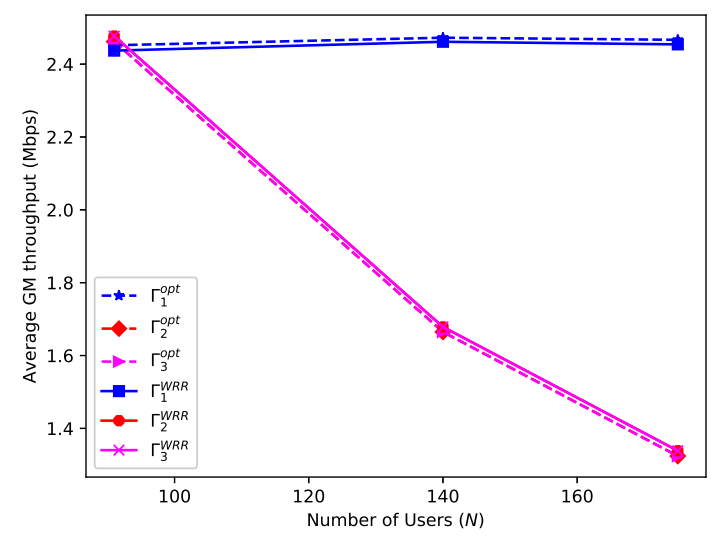

Figure 2.6: Comparison of the GM throughput with the optimal scheduler and with WRR as a function of $N_{2}=N_{3}=N$ for the three-operator system

\subsection{Conclusions}

We have proposed a snapshot model for evaluating the performance of SR on the downlink when we enforce equal gain and compared its performance with the benchmark where each operator works independently and only accept its own users. This model jointly optimizes UA and US. Through extensive computations for different systems (e.g., 2 operators, 3 operators, different topologies), we showed that the gains are significant. We then proposed a weighted round robin (WRR) heuristic scheduler to replace the legacy RR scheduler in all the BTSs and showed that it is quasi optimal in scenarios with 2 and 3 operators. SR can bring significant gains with little associated cost even when the BTSs of the different operators are co-located as long as the sectors are not aligned. The gains in sum throughput can be as large as $29 \%$ for the case with 2 operators and $43 \%$ for the case with 3 operators. 


\section{Chapter 3}

\section{Smart Roaming : Dynamic Case}

In this chapter we,

- We extend our analysis to dynamic settings

- Show that a simple scheme based on free UA and RR scheduling yield unacceptable cross subsidies and creates no operator discrimination.

- Propose two online schemes to implement SR that minimize cross subsidy and improve the performance

\subsection{Introduction}

The results of the static study in Chapter 2 are indicative of the potential of smart roaming, its true performance can only be determined within a dynamic setting. Therefore, we perform a thorough study in a dynamic setting where users arrive to the system according to a random process and stay in the system until they are fully served. The challenge is to implement an online scheme that offers the benefits seen in the static case whilst being practical. 


\subsection{Online Case}

As mentioned in Chapter 1, smart roaming can be implemented with a combination of UA and US. Typically US is local to a sector and does not require information from other sectors. UA schemes can be network centric or device-centric. Without loss of generality, we will assume a device-centric UA. In that case, the association decisions are made by the users themselves, using their channel state information and possible other information about the network broadcasted by the BTSs. A popular UA scheme is based on Max-SINR, i.e., a user associates with the sector yielding the highest SINR. However, it was shown in [24] that restricting the UA decision to only physical layer measurements is sub-optimal. A UA scheme that takes the load on each sector into account performs much better. In that case, each BTS/sector needs to broadcast load information in its beacon.

In the following, we describe the UA and US schemes that we use for our "No Roaming (NR)" benchmark where each network is operated independently. We also describe a scheme that we call "Full Roaming (FR)" which uses the same legacy scheduling as in NR and allows a "Free UA" whereby the users to select the best sector among all the sectors in the system irrespective of their operators. We will show that as expected FR yields tremendous cross-subsidies and hence we propose and study two smart roaming schemes, namely:

- Smart Roaming 1 (SR1) scheme, which uses a WRR scheduler at each sector along with "Free UA". The weights given to each type of users is calculated using the insights from the static case.

- Smart Roaming 2 (SR2) scheme, which uses the legacy RR scheduler in each sector and a coordinated user association via an online distributed algorithm aimed at load sharing.

While NR and FR do not require any coordination between the operators, the two schemes that we propose rely on some coordination between the operators. 
Without loss of generality, we describe the cooperation mechanism in a system with two operators where each operator has a C-RAN (cloud RAN), see Fig. 3.1. The CRANs cooperate by periodically sharing some information (the exact information and the periodicity depend on the scheme and will be described later).

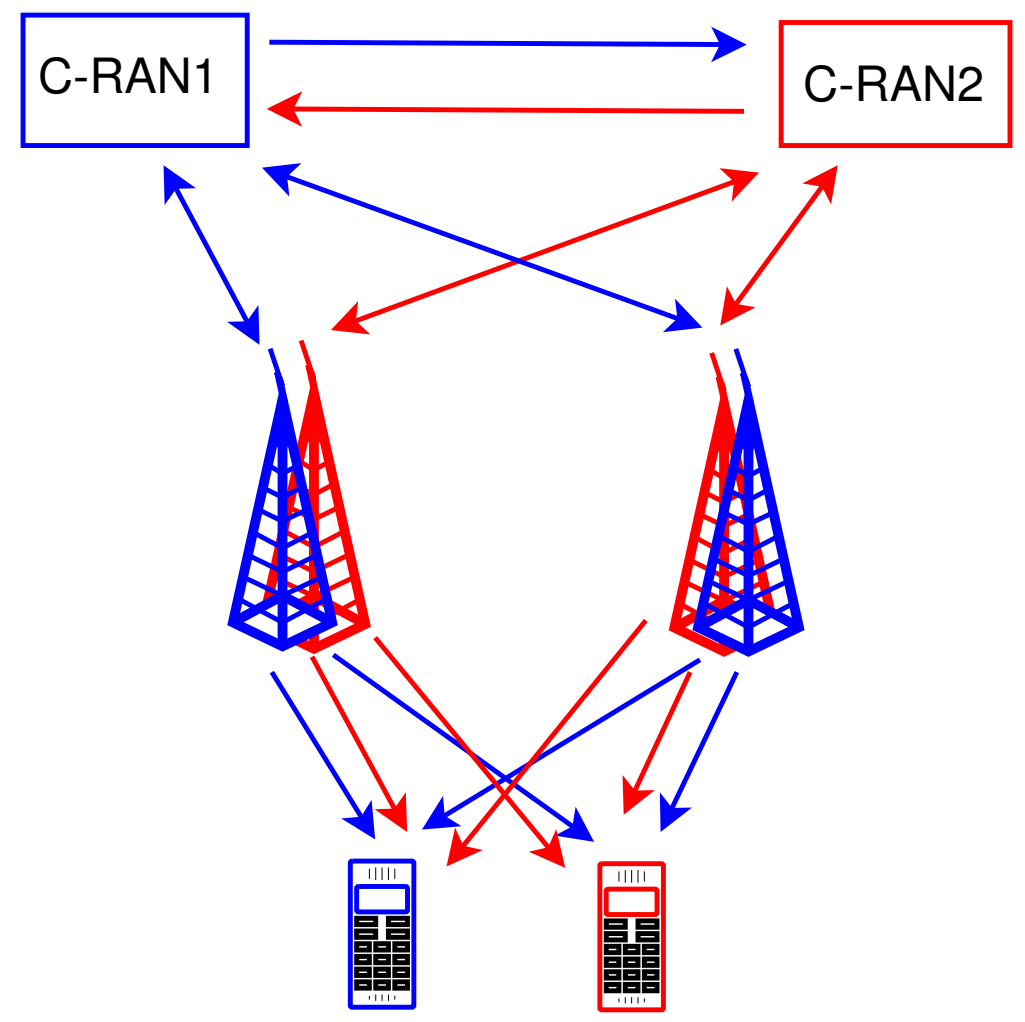

Figure 3.1: Network architecture

\subsubsection{No Roaming (NR)}

The benchmark remains the same as in the static case, i.e., each operator deals with its own users independently. In this scheme, US is RR and the UA rule is best rate, i.e., a user from operator $q$, arriving at time $t$, will select the sector $j^{*}$ in $\mathcal{Z}_{q}$ yielding the best 
rate, i.e.,

$$
j^{*}=\arg \max _{j \in \mathcal{Z}_{q}} \frac{R_{i, j}(t)}{n_{j}(t)+1}
$$

where $n_{j}(t)$ is the number of users associated with sector $j$ at time $t$. In order to compute $j^{*}$, the UE needs to be able to compute the link rates $R_{i, j}(t)$ 's and know the $n_{j}(t)$ 's. We assume that each sector broadcasts its $n_{j}(t)$ in its beacon.

\subsubsection{Full Roaming (FR)}

We consider a simple scheme called Full Roaming (FR), that enables the sharing of users without any constraints. In this scheme, the users are free to associate with any sector in the region irrespective of their operators. Similar to NR, sectors use a RR scheduling. "Free UA" is used whereby a user $i$, arriving at time $t$, will select the sector $j^{*} \in \mathcal{Z}$ yielding the best rate, i.e.:

$$
j^{*}=\arg \max _{j \in Z} \frac{R_{i, j}(t)}{n_{j}(t)+1}
$$

We will show that the performance seen by each operator is the same which creates unacceptable cross subsidies. Hence there is a need to define more elaborate schemes.

Recall that the equal gain formulation in the static case is performing well. However, it is not practical to translate it into an online scheme, as we cannot compute the GM throughput of each operator individually beforehand. We now describe the two schemes that we propose to minimize cross subsidies and provide good efficiency and operator discrimination.

\subsubsection{Smart Roaming 1 (SR1) scheme based on WRR}

The principle is simple: each sector uses a WRR scheduler to discriminate between users of operator 1 and operator 2 while UA is free for all in that each user can select the best sector in $\mathcal{Z}$, the difficulty being to estimate the rate that will be offered by each sector since this rate depends on WRR. We now describe in details the US and UA for SR1. 


\section{Revisiting WRR in a dynamic context}

Each sector $j$ will use the same WRR algorithm which allocates the same amount of time, $\alpha_{1 j}$, to users from operator 1 (there are $n_{1 j}$ such users) and the same amount of time, $\alpha_{2 j}$, to users of operator 2 (there are $n_{2 j}$ such users) and the ratio $c$ between $\alpha_{1 j}$ and $\alpha_{2 j}$ can be computed using Eq. (2.22). Note that this ratio is a function of quantities that rarely change with time, i.e., the number of sectors and the number of sub-channels per operator and quantities that vary, i.e., the number of users per operator. We propose that the computation of the ratio be done by the C-RANs which will periodically exchange an estimate of their number of users, i.e., the number of users averaged over the last one minute. Then, each C-RAN will compute the ratio and send the latest value to its BTSs every minute.

\section{User association for SR1}

Upon arrival, a user $i$ will select the sector that delivers the best rate irrespective of its operator. To compute the rate it will get in sector $j$, user $i$ of operator $q$ needs to know $c$, $n_{1 j}$ and $n_{2 j}$ at the time it arrives (say time $t$ ). This information is sent in the beacon of sector $j$. Given this information, user $i$ can compute $\alpha_{q j}$ assuming it joins $j$ and hence the rate it would receive if it associates with $j$.

\subsubsection{Smart Roaming 2 (SR2) scheme based on load sharing}

This scheme was designed for the two-operator case, with the a priori of keeping the scheduler unchanged, i.e., every sector performs RR over all its associated users irrespective of their operator. Hence, the burden of smart roaming without cross-subsidy is totally on the UA. The principle is that users can choose the best sector irrespective of their operator as long as the loads brought to each operator by the other one are about equal. We believe that such a scheme, if it performs well, might be easier to implement because it does not impact the scheduling. 
Specifically, we define load as the amount of bits an operator has transmitted on behalf of users of the other operator. Let $L_{q}(t)$ be the total number of bits received by all users of operator $q$ till time $t, U_{q}(t)$ be the total number of bits transmitted to all users associated with sectors in $\mathcal{Z}_{q}$ till time $t, D_{q q^{\prime}}$ be the number of bits received by users belonging to operator $q \in\{1,2\}$ from operator $q^{\prime} \in\{1,2\}$ till time $t$. We have $\forall q, q^{\prime} \in\{1,2\} q \neq q^{\prime}$

$$
\begin{aligned}
& U_{q}(t)=D_{q q}(t)+D_{q^{\prime} q}(t) \\
& L_{q}(t)=D_{q q}(t)+D_{q q^{\prime}}(t)
\end{aligned}
$$

Let the load surplus at operator $q$ at time $t$ be $S_{q}(t)$ :

$$
\begin{gathered}
S_{q}(t)=U_{q}(t)-L_{q}(t)=D_{q^{\prime} q}-D_{q q^{\prime}} \\
S_{1}(k)=-S_{2}(k)
\end{gathered}
$$

We now discuss a simple association rule that aims at keeping the surplus low over time and an adaptive algorithm to update network wide parameters that are used in this association rule.

\section{Association Rule}

The association rule followed by users of operator 1 is very simple, if a user $u$ arrives for operator 1 in frame $t$, then it will join operator 2 if and only if $R_{u}^{2}(t) \geq R_{u}^{1}(t)\left(1+\Delta_{1}\right)$ where $R_{u}^{1}$ (resp. $R_{u}^{2}$ ) is the highest rate $u$ can get from a sector in $\mathcal{Z}_{1}$ at time $t$ (resp. $\mathcal{Z}_{2}$ ), otherwise if will join operator 1 . Operator 2 will accept all users coming from operator 1 since they follow this rule. Similarly, the decision rule followed by user $v$ of operator 2 is very simple, in it will join operator 1 if and only if $R_{v}^{1}(t) \geq R_{v}^{2}(t)\left(1+\Delta_{2}\right)$, otherwise if will join operator 2 .

We now propose an algorithm to periodically (e.g., every minute) update the value of $\Delta_{1}$ at C-RAN1 (a similar algorithm is run at CRAN2 to update $\Delta_{2}$ ). 


\section{Updating parameters $\Delta_{1}$ and $\Delta_{2}$ at C-RAN1}

We describe the algorithm used by C-RAN1 to update $\Delta_{1}$ (there is a similar algorithm in C-RAN2 to update $\Delta_{2}$ ). This update mechanism is inspired by TCP, i.e., it does additive increase and multiplicative decrease. Let $S_{1}(k)$ be the surplus in the $k^{\text {th }}$ frame ${ }^{1}$ for operator 1. Note that C-RAN1 can compute the surplus by measuring $D_{21}$ and obtaining $D_{12}$ from C-RAN2 (and similarly for C-RAN2). Let the cumulative average surplus after $T$ minutes be $\Lambda(T)$

$$
\Lambda(T)=\sum_{l=1}^{6000 T} S_{1}(l)
$$

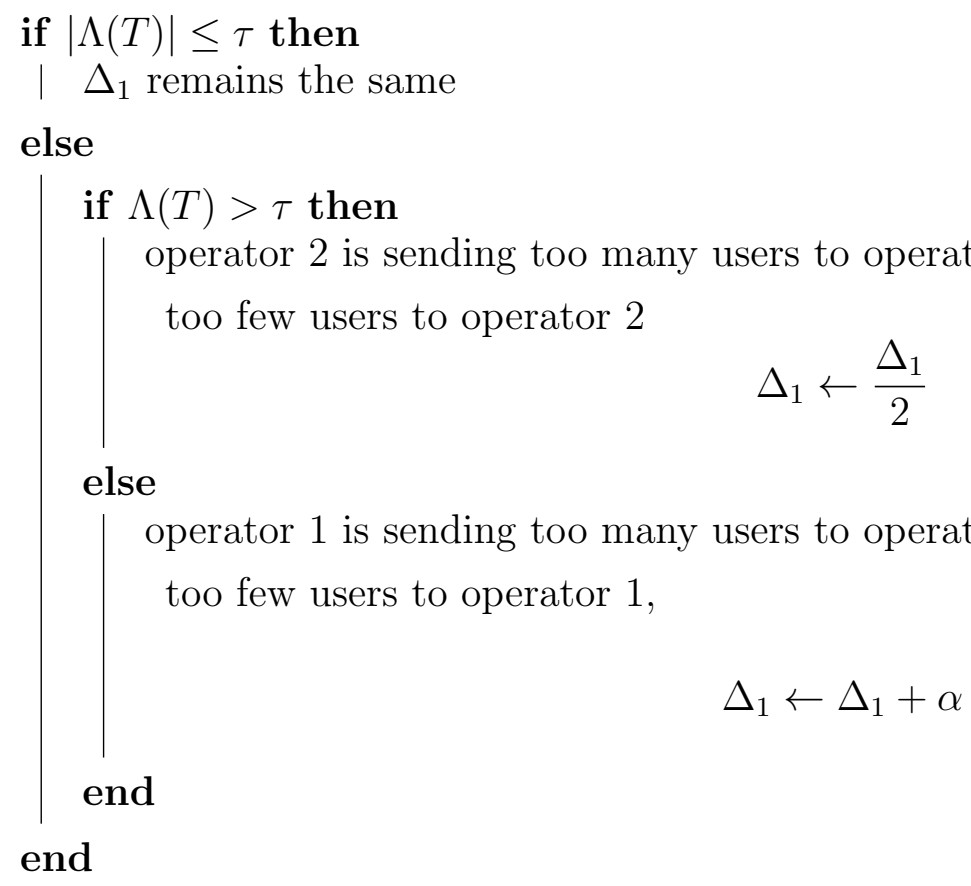$$
\Delta_{1} \leftarrow \frac{\Delta_{1}}{2}
$$

else too few users to operator 1 ,

$$
\Delta_{1} \leftarrow \Delta_{1}+\alpha
$$

end

end

operator 1 is sending too many users to operator 2 and operator 2 is sending

Algorithm 1: Updating $\Delta_{1}$ at C-RAN1

Essentially, if $|\Lambda(T)|$ is small with respect to a threshold $\tau>0, \Delta_{1}$ remains unchanged, otherwise if it is large and positive, then $\Delta_{1}$ is decreased by a factor of 2 and if it is large and negative, it is increased by an additive factor $\alpha>0$. The algorithm is given in Fig. 1,

\footnotetext{
${ }^{1}$ Scheduling is done every frame where a frame is $10 \mathrm{~ms}$.
} 
it has 2 input parameters $\tau>0$ (usually a large value) and $\alpha>0$ and we consider it at the beginning of the $(T+1)^{t h}$ minute. We start with $\Delta_{1}=\Delta_{2}=0$ at $t=0$.

We have tried simulations with $\alpha=0.5$ and 0.25 and found that the results were comparable, hence we use $\alpha=0.5$ for all the reported results. We ran the algorithm on many simulations and found that the choice of $\tau$ has a significant impact on the performance and that a value that is good for a simulation might be bad for another. Hence, we propose a method to obtain a good value of $\tau$ by adjusting it periodically, i.e., every 10 minutes, hence becomes $\tau(t)$.

\section{Updating threshold $\tau$}

Care must be taken to select $\tau$ appropriately. If a large value of $\tau$ is chosen, then our scheme SR2 behaves like FR with no change in parameters $\Delta_{1}$ and $\Delta_{2}$. If $\tau$ is too small, there will be no user exchange. A good value of $\tau$ should allow each operator to be in surplus from time to time, so we begin by choosing a very large value for $\tau$ (example $3 \times 10^{7}$ ). To adjust the value of $\tau$, we consider a jumping window of 10 minutes. The algorithm has one input parameter $\epsilon=0.1$. We compute the cumulative surplus over the 10 minutes and update $\tau$ as described in Algorithm 2.

\subsubsection{Numerical Results}

\section{The two-operator case}

In this section, we compare the performance of our two smart roaming schemes, SR1 and SR2 with FR and NR for two traffic scenarios in the case of a system with two operators where the BTSs are co-located and $\theta=60$ degrees. For both scenarios, we assume users of operator $q$ arrive according to a Poisson process of rate $\lambda_{q}$ users per second, are uniformly distributed over the region of interest and leave the system after being completely served. In Scenario 1, each user stays for a random time, sampled from an exponential distribution with mean $1 / \mu=60 \mathrm{sec}$. In that case, the natural performance metric for a user $u$ is the 
if Cumulative Surplus has not changed sign since last 10 minute period then The threshold is too large, reduce $\tau$

$$
\tau(t) \leftarrow \frac{\tau(t)}{2}
$$

\section{else \\ Refresh Window}

The threshold can be increased to allow more user exchanges

$$
\tau(t) \leftarrow \tau(t) \times(1+\epsilon)
$$

end

Refresh Window

\section{Algorithm 2: Updating $\tau$ at C-RAN1}

average download rate $\bar{\eta}_{u}$ seen during its sojourn time and the system metric for operator $q$ is the average download rate over all its users. This scenario could be used to model situations where users stream a video of a certain time duration. In this scenario, higher throughput to a user translates to a better quality of service, but the amount of time the user spends in the system is independent of the allocated throughput. In Scenario 2, each user downloads a fixed file of size $10 \mathrm{MB}$, and leaves the system after it has downloaded the file. The natural performance metric for a user $u$ is the delay to download the file and the system metric for operator $q$ is the average delay over all its users. All the results below are given with a $5 \%$ confidence interval. We consider the system corresponding to Cases given in Section 2.3.2. We show results in Figures 3.2-3.4 as a function of $\lambda_{2}$, the rate of arrival of users of operator 2 , while fixing $\lambda_{1}$, the rate of arrival of users of operator 1 .

We begin by comparing the performance of NR and FR for Scenario 1 in Figure 3.2 and 3.3 for case 1 and case 2 respectively where we label a curve by the corresponding operator (Op1 or Op2) and the scheme. We see that FR does not provide any operator discrimination, i.e., both operators get the same performance. Hence, FR yields unacceptable cross subsidies and for certain values of the parameters, it performs worst than NR for one of the operators, e.g., in Figure 3.3a for $\lambda_{2} \geq 1.75$ the performance of operator 1 with FR is 
significantly lower than the performance with NR. In scenario 2, we observe similar trends (see Figure 3.5a), where again FR leads to unacceptable cross subsidies. Thus a simple scheme of free UA combined with the legacy RR scheduling does not perform adequately.

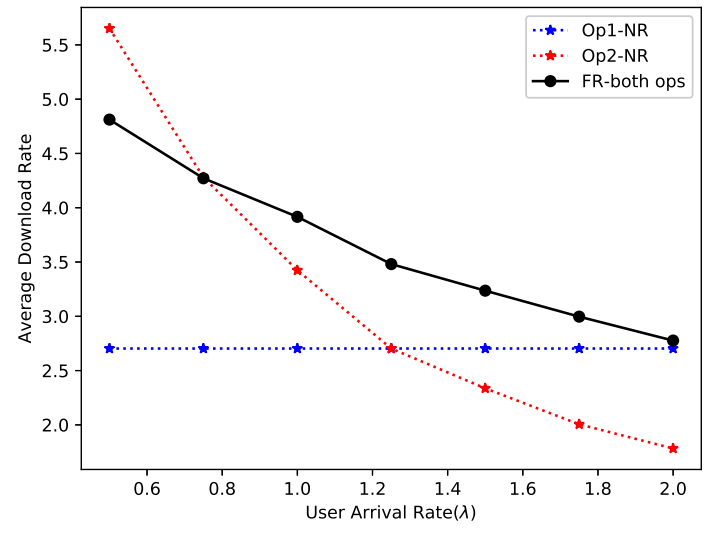

(a) Comparison between NR and FR

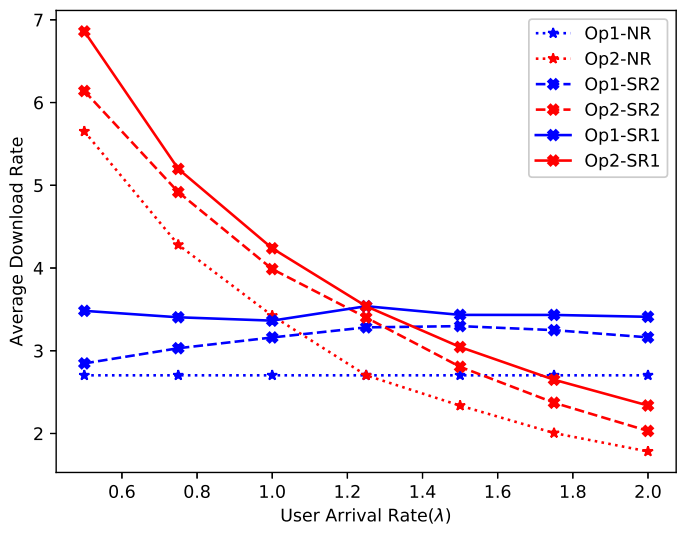

(b) Comparison between SR1 and SR2

Figure 3.2: Scenario 1: Average download rate as a function of $\lambda_{2}, \lambda_{1}=1.25$

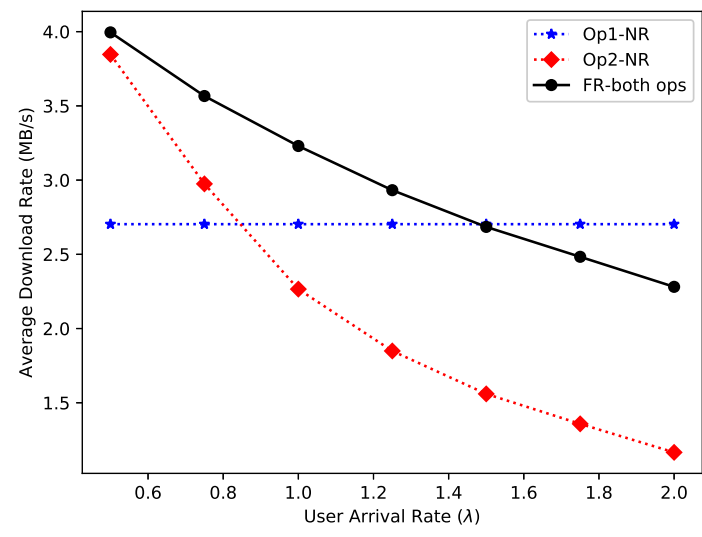

(a) Comparison between NR and FR

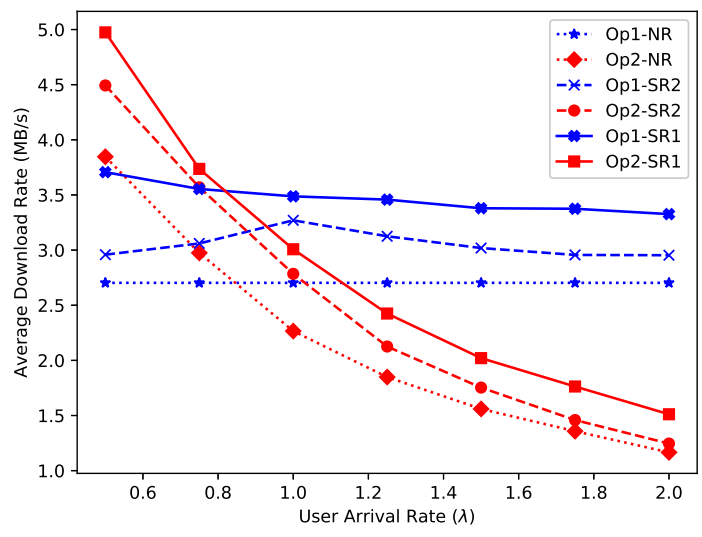

(b) Comparison between SR1 and SR2

Figure 3.3: Scenario 1: Average download rate as a function of $\lambda_{2}, \lambda_{1}=1.25$ 
The ideal behavior is where there is operator discrimination, no cross subsidies and the relative performance of operators remain the same, i.e., in the case of Scenario 1, we would like a upward vertical shift of the two curves corresponding to NR (higher rates) and in the case of scenario 2, a downward vertical shift (lower delay). We can achieve this kind of behavior with the proposed schemes SR1 and SR2 as shown in Figures 3.2b ,3.3b, 3.4b and 3.5b. We see that SR1 yields better gain than SR2. For Scenario 1, the gains in sum throughput for SR1 range between 20-30\% and between 15-23\% for SR2. Particularly we see that using SR1 the performance of operator 1 is near constant, whereas it is not so in SR2.

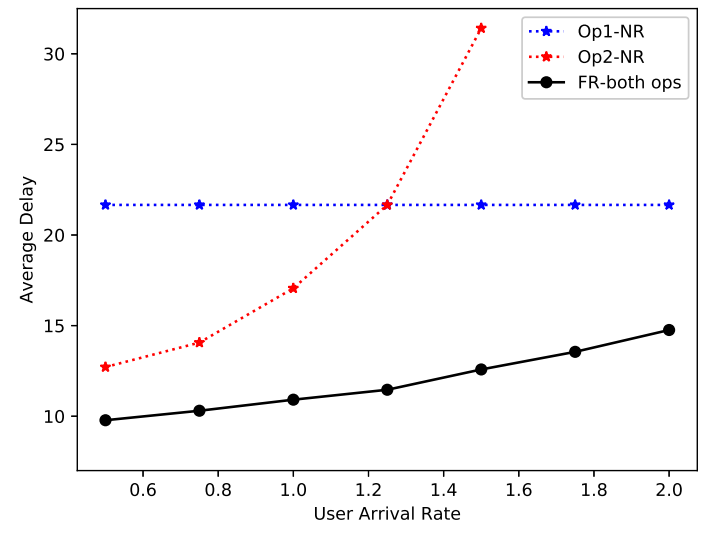

(a) Comparison between NR and FR

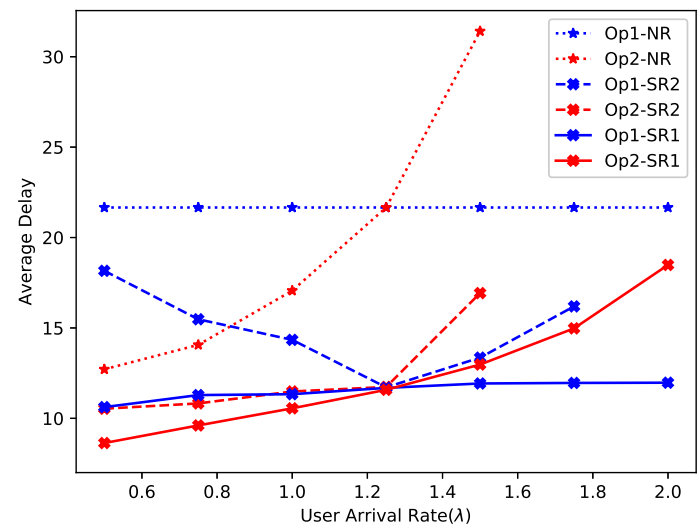

(b) Comparison between SR1 and SR2

Figure 3.4: Scenario 2: Average Per user delay, $\lambda_{1}=1.25$

We see that SR1 also performs much better in Scenario 2. Since we only control UA in SR2, the delay is not constant for operator 1 and the performance decreases for larger $\lambda_{2}$, whereas using SR1 yields larger gains and near constant delay for operator 1 even for larger $\lambda_{2}$. Thus we can see that the scheme (SR1) based on WRR scheduler outperforms SR2 which only operates on the UA. The gains in delay are very large which indicates that SR1 works very well in both traffic scenarios. 


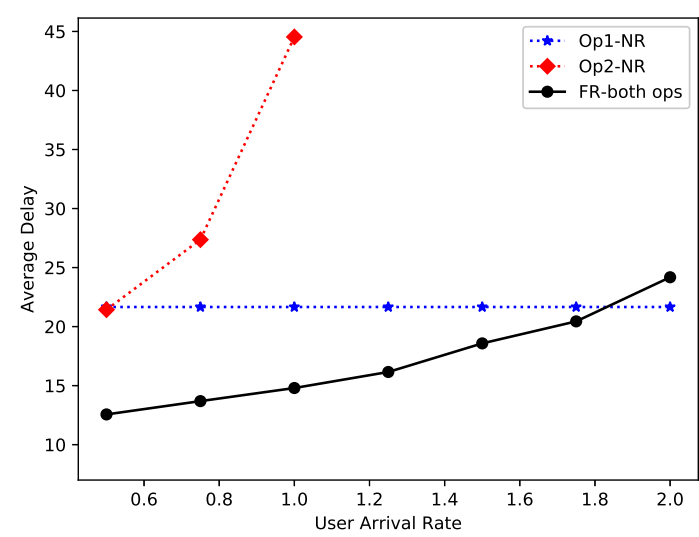

(a) Comparison between NR and FR

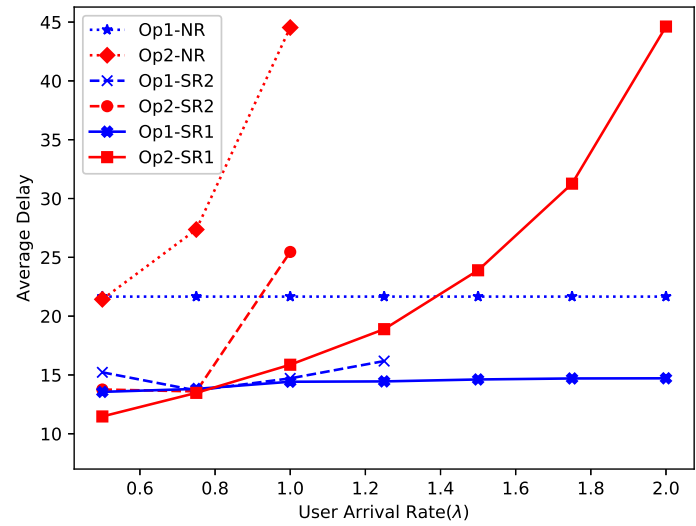

(b) Comparison between SR1 and SR2

Figure 3.5: Scenario 2: Average Per user delay, $\lambda_{1}=1.25$

We now move onto the three operator case.

\section{The three-operator case}

We also consider a system with three operators and focus on SR1. We consider the colocated topology with $\psi=40$ (see Section 2.3.2). We compare the results as a function of $\lambda\left(\lambda_{2}=\lambda_{3}=\lambda\right)$, while fixing $\lambda_{1}$ for NR, SR1 and FR in Figures 3.6a and 3.6b for Scenario 1 and Scenario 2 respectively.

Again we see that FR yields the same performance for the 3 operators and hence, severe cross subsidies in some cases. For example, operator 1 is penalized at higher $\lambda$. Similar to the static setting, we see that compared to the two operator system, we get higher gains in performance for the three operator system. Note that since both operators 2 and 3 have identical parameters, they have the same performance. There is a significant gain in sum throughput of $33-42 \%$ for Scenario 1 and the average delay in Scenario 2 is decreased by a factor of almost 2 for all operators. Similar to the two operator system, the average per user rate and delay for operator 1 in Scenario 1 and Scenario 2 respectively remains near constant even for larger values of $\lambda$. 


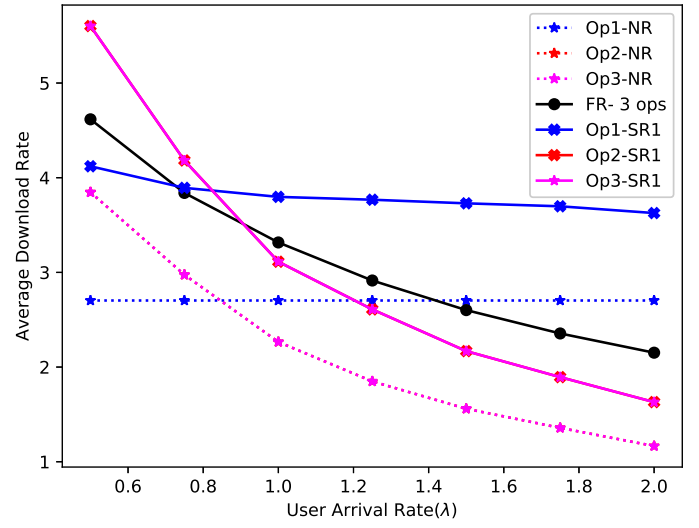

(a) Scenario 1

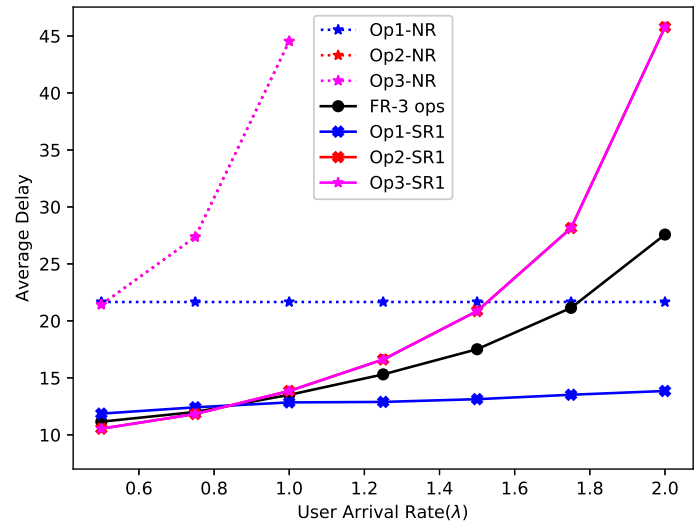

(b) Scenario 2

Figure 3.6: Three operator case

\subsection{Conclusion}

In conclusion, We studied dynamic setting and proposed and evaluated two schemes to implement SR online for 2 and 3 operator case. We discussed, for each scheme the information to be exchanged by the operators. We showed that a simple scheme based on free UA and RR yields cross subsidy and that our proposed schemes minimizes cross subsidy and ensures operator discrimination. We showed that a free UA and a WRR scheduler in each BTS performs better than a scheme just based on UA with legacy RR scheduler. 


\section{Chapter 4}

\section{Conclusion and Future Work}

\subsection{Contributions}

We have studied smart roaming (SR), a cooperation scheme between operators operating in the same region, as a means to improve spectrum usage efficiency. We formulated a snapshot (static) model for evaluating the potential performance of SR for systems with two and three operators and different topologies. We have shown that SR leads to significant gains (29\% in sum throughput for the case with two operators and $43 \%$ for the case with three operators) respect to the case where operators do not collaborate, even when BTSs are co-located, as long as their sectors are not aligned. We extracted from the numerical results of the snapshot model a weighted round robin (WRR) heuristic scheduler to use in all the BTSs and showed that it is quasi optimal.

We then moved to the dynamic case and showed that a simple and "natural" scheme based on a "free UA" where each user can select the best BTS irrespective of her operator combined to a legacy RR scheduler at all BTSs does not perform well in that it yields unacceptable cross-subsidies. Therefore, we proposed two schemes to implement SR online for the two operator case. We discussed the information to be exchanged between operators for each of the scheme and compared the gains that both the schemes yield. The main results is that SR1 (i.e., the one based on the WRR scheduling) outperforms SR2 and that 
the gains of SR1 over the No Roaming case are significant, for example for the case of scenario 2 with three operators, the mean delay could be more than halved. One of the nice attributes of SR1 is that it can be generalized to the case of more than two operators. One of its shortcomings is that it requires a change of scheduling. Despite our attempts, we could not design a scheme that keeps the legacy RR scheduler and performs as well as a scheme that replaces RR with WRR in all BTSs. It might not be easy to convince operators to use SR1 since they are typically reluctant to modify their legacy RR scheduler. However, governing bodies like FCC could mandate it in certain spectrum bands to improve the usage efficiency of these bands since the performance gains are significant.

\subsection{Future Work}

In this study, we restricted ourselves to the downlink transmissions. An equally important problem would be to investigate the performance gains of smart roaming on the uplink. It would also be interesting to study SR under a scenario where operators have different hot spots. These are for future studies. 


\section{References}

[1] Baron for ampl. https://ampl.com/products/solvers/solvers-we-sell/baron/.

[2] Canadian cellular towers map. https://www.ertyu.org/steven_nikkel/ cancellsites.html. Accessed: 2017-01-30.

[3] Minos for ampl. https://ampl.com/products/solvers/solvers-we-sell/minos/.

[4] 3GPP TS 23.251. Network sharing; architecture and functional description. Available: http://www.3gpp.org/ftp/Specs/html-info/23251.html.

[5] 3GPP-TSG-RAN-WG1. Evolved universal terrestrial radio access (e-utra), 3GPP, Tech. Rep. TR 36.814, 2010.

[6] J. G. Andrews, S. Buzzi, W. Choi, S. V. Hanly, A. Lozano, A. C. K. Soong, and J. C. Zhang. What will $5 \mathrm{~g}$ be? IEEE Journal on Selected Areas in Communications, 32(6):1065-1082, June 2014.

[7] Alex Apostolidis, Luis Campoy Cervera, Konstantinos Chatzikokolakis, Karl-Josef Friederichs, Tim Irnich, Konstantinos Koufos, Jonas Kronander, Jian Luo, Eiman Mohyeldin, Pedro Olmos, Thomas Rosowski, Hans Schotten, Bikramjit Singh, Miurel Tercero, Olav Tirkkonen, and Mikko A. Uusitalo. Intermediate description of the spectrum needs and usage principles, 082013.

[8] D. Fooladivanda and C. Rosenberg. Joint resource allocation and user association for heterogeneous wireless cellular networks. IEEE Transactions on Wireless Communications, 12(1):248-257, January 2013. 
[9] D. Fooladivanda and C. Rosenberg. Why user swapping could be the best coordination mechanism in a cellular network? In 2013 IEEE Global Communications Conference (GLOBECOM), pages 4895-4901, Dec 2013.

[10] T. Frisanco, P. Tafertshofer, P. Lurin, and R. Ang. Infrastructure sharing and shared operations for mobile network operators: From a deployment and operations view. In 2008 IEEE International Conference on Communications, pages 2193-2200, May 2008 .

[11] C. Gabriel. Swedish operators take ran sharing into lte, rethink wireless, April, 2009. Available: http://www.telecomseurope.net/content/swedish-operatorstake-ran-sharing-lte.

[12] F. Graziosi and F. Santucci. A general correlation model for shadow fading in mobile radio systems. IEEE Communications Letters, 6(3):102-104, March 2002.

[13] E. A. Jorswieck, L. Badia, T. Fahldieck, D. Gesbert, S. Gustafsson, M. Haardt, K. Ho, E. Karipidis, A. Kortke, E. G. Larsson, H. Mark, M. Nawrocki, R. Piesiewicz, F. Rmer, M. Schubert, J. Sykora, P. Trommelen, B. van den Ende, and M. Zorzi. Resource sharing in wireless networks: The saphyre approach. In 2010 Future Network Mobile Summit, pages 1-8, June 2010.

[14] J. Kibida, B. Galkin, and L. A. DaSilva. Modelling multi-operator base station deployment patterns in cellular networks. IEEE Transactions on Mobile Computing, 15(12):3087-3099, Dec 2016.

[15] A. Kumar and C. Rosenberg. Energy and throughput trade-offs in cellular networks using base station switching. IEEE Transactions on Mobile Computing, 15(2):364-376, Feb 2016.

[16] E. G. Larsson, O. Edfors, F. Tufvesson, and T. L. Marzetta. Massive mimo for next generation wireless systems. IEEE Communications Magazine, 52(2):186-195, February 2014. 
[17] Djamal-Eddine Meddour, Tinku Rasheed, and Yvon Gourhant. On the role of infrastructure sharing for mobile network operators in emerging markets. Comput. Netw., 55(7):1576-1591, May 2011.

[18] FCC Notice of Inquiry 10-237. Promoting more efficient use of spectrum through dynamic spectrum use technologies., Nov. 2010. Available: http://hraunfoss.fcc.gov/edocs public/attachmatch/FCC-10-198A1.pdf.

[19] Y. Ozcan and C. Rosenberg. Efficient loss-aware uplink scheduling. In 2018 IEEE Wireless Communications and Networking Conference (WCNC), pages 1-6, April 2018.

[20] J. S. Panchal, R. D. Yates, and M. M. Buddhikot. Mobile network resource sharing options: Performance comparisons. IEEE Transactions on Wireless Communications, 12(9):4470-4482, September 2013.

[21] R1-081957. Categorization of technical proposals for the phy layer of lte-a. Qualcomm Europe.

[22] T. S. Rappaport, S. Sun, R. Mayzus, H. Zhao, Y. Azar, K. Wang, G. N. Wong, J. K. Schulz, M. Samimi, and F. Gutierrez. Millimeter wave mobile communications for $5 \mathrm{~g}$ cellular: It will work! IEEE Access, 1:335-349, 2013.

[23] T. Sanguanpuak, S. Guruacharya, E. Hossain, and M. Latva-aho. Inter-operator infrastructure sharing: Trade-offs and market. In 2017 IEEE International Conference on Communications Workshops (ICC Workshops), pages 73-78, May 2017.

[24] Shaverdian, Ararat. A comparative study of resource allocation schemes in heterogeneous cellular networks on the downlink. Master's thesis, 2016.

[25] R. H. Tehrani, S. Vahid, D. Triantafyllopoulou, H. Lee, and K. Moessner. Licensed spectrum sharing schemes for mobile operators: A survey and outlook. IEEE Communications Surveys Tutorials, 18(4):2591-2623, Fourthquarter 2016. 